\title{
Electron-impact-ionization measurements using hyperfine-assisted state preparation of ground-state berylliumlike sulfur
}

\author{
M. Hahn, ${ }^{1, *}$ D. Bernhardt, ${ }^{2}$ M. Grieser, ${ }^{3}$ C. Krantz, ${ }^{3}$ M. Lestinsky, ${ }^{4}$ A. Müller, ${ }^{2}$ O. Novotný, ${ }^{1}$ \\ R. Repnow, ${ }^{3}$ S. Schippers, ${ }^{2}$ A. Wolf, ${ }^{3}$ and D. W. Savin ${ }^{1}$ \\ ${ }^{1}$ Columbia Astrophysics Laboratory, Columbia University, 550 West 120th Street, New York, New York 10027, USA \\ ${ }^{2}$ Institut für Atom- und Molekülphysik, Justus-Liebig-Universität Giessen, Leihgesterner Weg 217, 35392 Giessen, Germany \\ ${ }^{3}$ Max-Planck-Institut für Kernphysik, Saupfercheckweg 1, 69117 Heidelberg, Germany \\ ${ }^{4}$ GSI Helmholtzzentrum für Schwerionenforschung, Planckstr. 1, 64291 Darmstadt, Germany
}

(Received 10 February 2012; published 12 April 2012)

\begin{abstract}
We have measured electron impact ionization (EII) of the beryllium-like ion $\mathrm{S}^{12+}$. The use of an isotopically pure $A=33$ beam of $\mathrm{S}^{12+}$ ions is established as a method to eliminate all metastable levels, including the extremely long-lived metastable level $2 s 2 p^{3} P_{0}$, which, for this isotope, decays by hyperfine-induced radiative transitions. The energy dependence and absolute size of the EII cross section were measured from the resulting pure ground-state population at energies from below the threshold at $\approx 652.2 \mathrm{eV}$ up to $3000 \mathrm{eV}$. These data provide an experimental benchmark for theory.
\end{abstract}

DOI: 10.1103/PhysRevA.85.042713

PACS number(s): $34.80 . \mathrm{pp}$

\section{INTRODUCTION}

Accurate cross-section data for electron impact ionization (EII) are needed to interpret the spectra of collisionally ionized plasmas both in astrophysics [1,2] and in the laboratory [3]. Spectroscopic diagnostics of such plasmas rely on accurate ion balance calculations to determine the electron temperature, electron density, and elemental abundances [4-6]. These charge-state distributions are determined by the balance between EII and electron-ion recombination. Collisionally ionized plasmas are formed in stellar coronae, supernova remnants, galaxies, and the intracluster medium of galaxy clusters, as well as in tokamaks and other laboratory plasmas. Accurate EII data for every isoelectronic sequence are needed to reliably interpret spectra from all these systems.

Currently, only a small amount of the needed atomic amount has been obtained experimentally and most come from theory $[1,2]$. EII theory is complicated by the need to account for two outgoing electrons in the continuum and typically also by the complex atomic structure of the initial and final systems. Approximations are needed in order to make the theory tractable, but the associated uncertainties in the calculated cross sections are difficult to assess. In this situation accurate experimental benchmarks are vitally required in order to be able to judge the quality of the various theoretical approaches.

Obtaining the necessary data is particularly difficult in the case of beryllium-like ions because measurements almost always involve a large population of ions in metastable levels. Essentially all production methods generate Be-like ions with a large fraction in excited states. The triplet states mostly cascade down to the $2 s 2 p^{3} P_{0,1,2}$ levels. Compared to the $2 s^{2}{ }^{1} S_{0}$ ground state, often more than $50 \%$ of the ions are in the $2 s 2 p^{3} P$ levels. Of these the ${ }^{3} P_{0}$ level is especially troublesome because it can only decay by forbidden two-photon transitions combining an electric and magnetic dipole decay (E1M1), giving it a lifetime of the order of days to years $[7,8]$.

\footnotetext{
*mh2451@ columbia.edu
}

Because Be-like ion beams are so strongly affected by metastable contamination, much work has focused on determining the metastable fraction in the various experimental studies. This can then be taken into account in the interpretation of the results and in comparisons to theoretical calculations. For resonant processes, such as dielectronic recombination [9,10] and photoionization [11], resonances associated with the metastable ${ }^{3} P_{J}$ levels can be identified, allowing the metastable fraction to be inferred using theoretical calculations as a guide. Such methods, though, are not an independent test of theory.

For EII studies, an indirect method for measuring the ground-state cross section is to vary the metastable fraction in the beam and extrapolate the ground-state cross section [12]. However, it is difficult to reproducibly manipulate the metastable fraction. One can also determine the metastable fraction directly by passing the beam through a helium gas cell as a function of the gas density [13]. The ground-state and ${ }^{3} P$ levels have different electron capture cross sections so the attenuation rates for the ground-state and metastable components of the beam are different, allowing the metastable fraction to be inferred. However, it is not possible to quantify the population of the various $J$ levels. This method is also limited to low-charge states where the ground-state and metastable capture cross sections differ significantly [13]. Thus, for EII measurements on most Be-like ions it is not possible to directly measure the metastable fraction. Theoretical calculations involving a sum of ground-state and metastable cross sections can be compared to the experimental results to help estimate the metastable fraction [14], but such methods cannot be used to test theory.

We are aware of only one previous method developed to generate pure beams of Be-like ions. That approach was used for performing electron capture measurements [15]. A Li-like ion beam was passed through a gas cell to generate a Be-like beam through electron capture. Due to conservation of energy, the kinetic energies of the resulting ion beams differed depending on the energy of the level into which the electron was captured. An energy analyzer was used to select out the desired electronic term. The selected ion beam was then passed 
through a second gas cell to measure the capture cross section. This method, however, could not separate out the different metastable ${ }^{3} P$ levels and has only been used for relative cross section measurements.

Here we have performed ion storage ring measurements of $\mathrm{S}^{12+}$. Sulfur is cosmically abundant. $\mathrm{S}^{12+}$ is commonly observed in solar active regions and provides many useful diagnostics for solar physics [16]. For our measurements we used the ${ }^{33} \mathrm{~S}$ isotope, which has a nuclear spin, whereas the more abundant ${ }^{32} \mathrm{~S}$ does not. The resulting hyperfine interaction induces a mixing of the ${ }^{3} P_{1}$ and ${ }^{3} P_{0}$ levels, decreasing the lifetime of the ${ }^{3} P_{0}$ level [17]. In the absence of such mixing, the ${ }^{3} P_{0}$ level has a lifetime of $6.3 \times 10^{6} \mathrm{~s}$ [7]. But the lifetime for ${ }^{33} \mathrm{~S}^{12+}$ has been calculated to be $10.7 \mathrm{~s}[18,19]$ and measured in connection with the present work to be $10.4 \pm 0.5 \mathrm{~s}$, where the uncertainty is given at the $1 \sigma$ level [20]. The ${ }^{3} P_{1}$ and ${ }^{3} P_{2}$ levels decay radiatively with the much shorter lifetimes of $9 \times 10^{-7}$ and $6.9 \times 10^{-2} \mathrm{~s}$, respectively [16]. Performing the experiments with an ion storage ring therefore allowed the ions to be stored long enough before data were collected for all of the metastable levels to radiatively relax to the ground state. Hence, our results provide unambiguous measurements of EII from the ground state of a Be-like ion.

\section{EXPERIMENTAL METHOD}

The experiments were performed using the TSR heavy-ion storage ring at the Max-Planck-Institut für Kernphysik in Heidelberg, Germany (Fig. 1). The procedures used here closely follow those described in Refs. [20-23]. The measurements proceeded in two steps. First, the ${ }^{33} S^{12+}$ ions were injected with an energy of about $56 \mathrm{MeV}$ into the TSR, where they were merged with two electron beams, called the Cooler and the Target, each in a different section of the ring. Initially both electron beams were operated in modes designed to reduce the energy spread of the stored ions, commonly called electron cooling. The cooling cycle lasted $30 \mathrm{~s}$, during which time metastable levels could radiatively decay to the ground state. The initial fraction of ions in the ${ }^{3} P_{0}$ level has been estimated to be $<10 \%$ [20]. The associated lifetime of $\approx 10.4 \mathrm{~s}$ [20] is orders of magnitude longer than that of any of the other

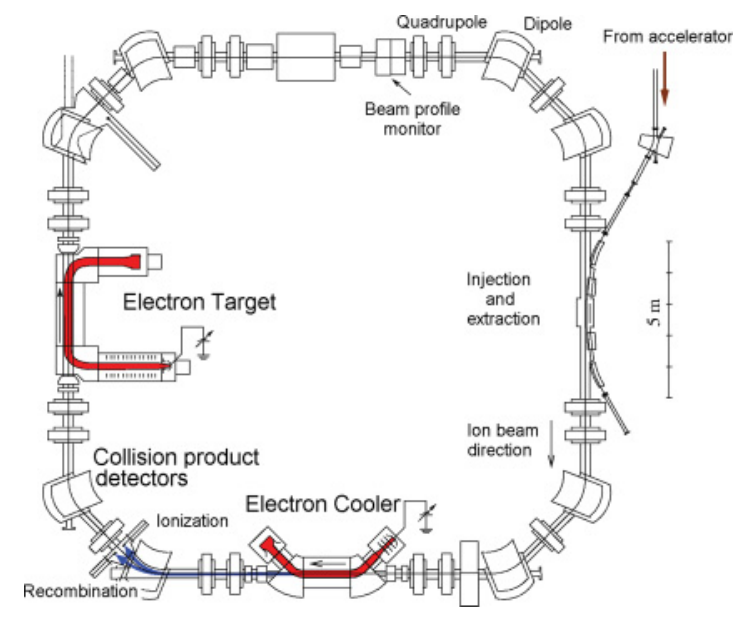

FIG. 1. (Color online) Diagram of the TSR heavy-ion storage ring. See text for details. metastable levels, resulting in a metastable fraction in the beam after $30 \mathrm{~s}$ of $<0.5 \%$. Collisions can also excite ions from the ground state into a metastable level throughout the measurement cycle. Based on the collision strengths [16] and typical electron and residual gas densities, we estimate that the excitation rate to the ${ }^{3} P_{0}$ level was less than $10^{-4} \mathrm{~s}^{-1}$. These collisional excitations were balanced by the hyperfine-induced transition rate of $\sim 0.1 \mathrm{~s}^{-1}$, which implies a collisionally induced steady-state metastable fraction of $\lesssim 0.1 \%$.

After cooling, electron-ion collisions were studied by scanning the Cooler electron energy, while the Target energy was maintained at cooling. Ions that underwent ionization or recombination while passing through the Cooler electron beam were deflected by a dipole magnet downstream of the interaction section. Charged particle detectors intercepted and counted these product beams with an efficiency of nearly $100 \%[20,24]$. The background rate due to electron stripping off the residual gas was measured at a fixed reference energy in between each step of the measurement energy scan. For $E>1400 \mathrm{eV}$ the limited dynamic range of the high-voltage power supply used to scan the electron energy prevented the reference point from being set below the EII threshold. Consequently, the reference rate for $E>1400 \mathrm{eV}$ includes some counts due to EII. For these scans the background rate was inferred by combining the reference count rate with the EII cross section determined from the lower energy data.

The EII cross section $\sigma_{\mathrm{I}}$ was determined by normalizing the background subtracted count rate by the electron density and the number of stored ions in the interaction region. The electron density was determined from the measured current and known geometry of the Cooler electron beam. The estimated $1 \sigma$ uncertainty in the electron density is about 3\% [25]. The number of stored ions is proportional to the ion current, which was measured using a beam profile monitor (BPM) [26]. The BPM calibration depends on the residual gas pressure and electronic drifts and tends to vary on a time scale of a few hours. We periodically cross-calibrated the BPM with a dc transformer [27] using currents of up to $35 \mu \mathrm{A}$. The dc transformer has a stable calibration but cannot accurately measure the small, $\sim 1-10 \mu \mathrm{A}$, range of ion currents present during measurement. The estimated $1 \sigma$ uncertainty on $\sigma_{\text {I }}$ due to the ion current measurement is $\pm 15 \%$. This uncertainty only applies to the normalization and does not affect the shape of the cross section as a function of energy.

Distortions of $\sigma_{\mathrm{I}}$ due to pressure fluctuations during the measurement were corrected for using the recombination detector signal as a proxy for the pressure as described in Ref. [21]. Here, the magnitude of the correction below $1400 \mathrm{eV}$ was about a factor of 1.07 with a $1 \sigma$ uncertainty of about $\pm 1 \%$. Because our method of correcting for the pressure fluctuation requires that the reference energy be below the EII threshold, it could not be applied to data with $E>1400 \mathrm{eV}$. Thus, we estimate that there is an additional $\approx 7 \%$ systematic uncertainty in $\sigma_{\mathrm{I}}$ at these higher energies. Table I summarizes the various experimental uncertainties.

\section{RESULTS}

Figure 2 shows the EII cross section for $\mathrm{S}^{12+}$ forming $\mathrm{S}^{13+}$ over the experimental electron-ion collision energy range 
TABLE I. Sources of uncertainty.

\begin{tabular}{lc}
\hline \hline Source & Estimated $1 \sigma$ uncertainty \\
\hline Counting statistics & $1 \%$ \\
Ion current measurement & $15 \%$ \\
Electron density & $3 \%$ \\
Pressure fluctuations $^{\mathrm{a}}$ & $1 \%(7 \%)$ \\
Quadruature sum $^{2}$ & $15 \%(17 \%)$ \\
\hline \hline
\end{tabular}

${ }^{a}$ The $7 \%$ uncertainty applies only to data for $E>1400 \mathrm{eV}$, where the correction for pressure fluctuations could not be applied.

500-3000 eV. The measured cross section is essentially 0 below the threshold at $\approx 652.2 \mathrm{eV}$ [28] for direct ionization of one outer $2 s$ electron and rises steeply at higher energies until it reaches a maximum at $E \approx 1700 \mathrm{eV}$. At an even higher energy of $\approx 2400 \mathrm{eV}$ the cross section exhibits a step-like feature which appears on top of the smooth energy dependence due to direct ionization. This step is attributed to excitationautoionization (EA), a process that involves electron-impact excitation of at least one inner-shell electron to a multiply excited configuration which subsequently autoionizes. Excitation to $1 s 2 l^{3}$ configurations can occur above $\approx 2410 \mathrm{eV}$ and to $1 s 2 p^{2} 3 l$ configurations above $\approx 2785 \mathrm{eV}$ [1].

In Fig. 2 the $1 \sigma$ statistical uncertainties are indicated by error bars and the overall $1 \sigma$ systematic uncertainty is illustrated by dotted lines. The experimental cross section is compared to the theoretical cross section of Younger [29] used in ionization balance calculations [30,31] and to the distortedwave calculation of Dere [1]. Both theoretical cross sections consider the direct ionization channel and agree well with the measurement. The Dere [1] cross section additionally includes EA, and the predicted energy thresholds and magnitude are very close to the experimental results.

Agreement in magnitude to within the $15 \%$ systematic uncertainty is state of the art for ionization cross-section work. For example, we have found discrepancies of about $25 \%$ for

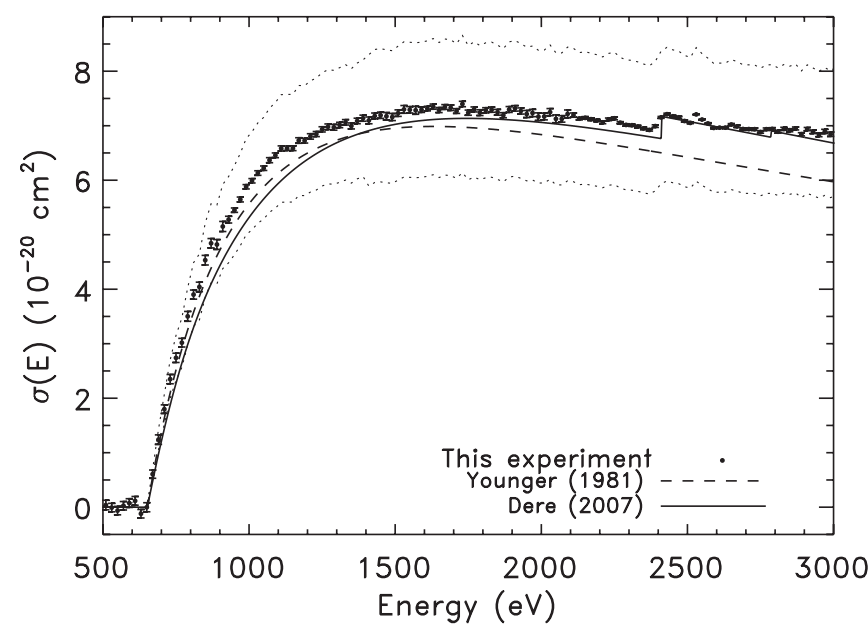

FIG. 2. EII cross section for $\mathrm{S}^{12+}$ forming $\mathrm{S}^{13+}$. Circles indicate experimental values and smaller error bars show the $1 \sigma$ statistical uncertainty. Dotted lines illustrate the $1 \sigma$ systematic uncertainty limits. Experimental results are compared to the theoretical cross section of Younger [29] and to the distorted-wave calculation of Dere [1]. other systems [22]. Theoretical calculations for Be-like ions predict that the metastable EII cross section can be 1.3 to 2 times larger than the ground-state cross section [13,29]. Thus, hyperfine-assisted state preparation to remove metastables is critical in order to provide a benchmark at the current high level of accuracy. We emphasize that the experimental systematic uncertainty is essentially a scaling factor and so the cross section's energy dependence is measured to an even greater accuracy than its magnitude.

\section{DISCUSSION}

The behavior of the cross section near threshold also supports the hypothesis that EA contributes to the cross section of $\mathrm{Fe}^{11+}$ and $\mathrm{Fe}^{12+}$ near the ionization threshold $[22,23]$. We found that the cross sections for $\mathrm{Fe}^{11+}$ and $\mathrm{Fe}^{12+}$ rose more rapidly near threshold than predicted by theory. Two physical effects could account for this discrepancy. These ions have a $3 s^{2} 3 p^{q}(q=2,3)$ configuration. One possibility is that a $3 s$ electron is collisionally excited to a state that relaxes through autoionization of a $3 p$ electron, a process that is not considered in the published theoretical calculations. The other possibility is that the enhancement is an artifact of the experiment. If a bound electron is collisionally excited to a high enough energy level, it could then be field ionized by the motional electric fields experienced by the ions as they travel through the TSR magnetic fields. A semiclassical estimate [32] showed that this could occur for excitations to $n \gtrsim 44$ for $\mathrm{Fe}^{11+}$ and $n \gtrsim 50$ for $\mathrm{Fe}^{12+}$. In the case of $\mathrm{S}^{12+}$, EA is not possible near the ionization threshold, but field ionization could still occur for excitations to $n \gtrsim 64$. However, we do not find any significant increase in the cross section near threshold. This suggests that it is EA, and not field ionization, that causes most of the enhancement in the earlier $\mathrm{Fe}^{11+}$ and $\mathrm{Fe}^{12+}$ measurements.

An alternative theoretical explanation for the discrepancies observed between theory and experiment is that the difference is caused by the form of the potential used in the distorted-wave calculations. Younger [29] used a form of the potential, known as the prior form, in which the incident and scattered electrons see an $N$-electron potential while the bound and ejected electrons are calculated in an $N-1$ potential [33], where $N$ is the number of electrons in the ion. Dere [1] used a different option, known as the post form, where all the scattering amplitudes are calculated for an $N-1$ electron potential $[34,35]$. For $\mathrm{S}^{12+}$ the two theory cross sections are similar, which suggests that the choice of potential makes little difference here. This is expected for multiply charged atomic ions since, for such ions, there is little difference between the $N$ and the $N-1$ potentials [36]. Thus, the form of potential used in the distorted-wave calculations seems unlikely to account for the observed discrepancies between theory and experiment near threshold.

In summary, we have developed a method for eliminating the metastable contributions from a beam of Be-like ions by using the hyperfine-induced decays combined with an ion storage ring. With this technique we have measured the EII cross section for $\mathrm{S}^{12+}$ from an essentially metastable-free ion beam. The experimental cross section was found to be in very good agreement with theoretical calculations. Thus, our experimental benchmark strengthens the confidence in 
the theoretical methods that are presently used in ion balance calculations for astrophysical and laboratory plasmas.

\section{ACKNOWLEDGMENTS}

We thank D.-H. Kwon and K. Dere for stimulating discussions. We appreciate the efficient support by the
MPIK accelerator and TSR groups during the beamtime. This work was supported in part by the NASA Astronomy and Physics Research and Analysis program and the NASA Solar Heliospheric Physics program. We also acknowledge financial support from the Max Planck Society, Germany, and from Deutsche Forschungsgemeinschaft (Contract No. Schi 378/8-1).
[1] K. P. Dere, Astron. Astrophys. 466, 771 (2007).

[2] T. R. Kallman and P. Palmeri, Rev. Mod. Phys. 79, 79 (2007).

[3] N. J. Peacock, M. G. O'Mullane, R. Barnsley, and M. Tarbutt, Can. J. Phys. 86, 277 (2008).

[4] N. S. Brickhouse, in Atomic Processes in Plasmas (Tenth), American Institute of Physics Conference Series, Vol. 381, edited by A. L. Osterheld and W. H. Goldstein (AIP, Melville, NY, 1996), pp. 31-38.

[5] E. Landi and M. Landini, Astron. Astrophys. 347, 401 (1999).

[6] P. Bryans, E. Landi, and D. W. Savin, Astrophys. J. 691, 1540 (2009).

[7] R. W. Schmieder, Phys. Rev. A 7, 1458 (1973).

[8] C. Laughlin, Phys. Lett. A 75, 199 (1980).

[9] M. Fogle, N. R. Badnell, P. Glans, S. D. Loch, S. Madzunkov, S. A. Abdel-Naby, M. S. Pindzola, and R. Schuch, Astron. Astrophys. 442, 757 (2005).

[10] D. W. Savin et al., Astrophys. J. 642, 1275 (2006).

[11] A. Müller, S. Schippers, R. A. Phaneuf, A. L. D. Kilcoyne, H. Bräuning, A. S. Schlachter, M. Lu, and B. M. McLaughlin, J. Phys. B 43, 225201 (2010).

[12] R. A. Falk, G. Stefani, R. Camilloni, G. H. Dunn, R. A. Phaneuf, D. C. Gregory, and D. H. Crandall, Phys. Rev. A 28, 91 (1983).

[13] M. Fogle et al., Astrophys. J. Suppl. Ser. 175, 543 (2008).

[14] S. D. Loch, M. Witthoeft, M. S. Pindzola, I. Bray, D. V. Fursa, M. Fogle, R. Schuch, P. Glans, C. P. Ballance, and D. C. Griffin, Phys. Rev. A 71, 012716 (2005).

[15] J. B. Greenwood, D. Burns, R. W. McCullough, J. Geddes, and H. B. Gilbody, J. Phys. B 29, 5867 (1996).

[16] E. Landi and A. K. Bhatia, At. Data Nucl. Data Tables 94, 1 (2008).

[17] W. R. Johnson, Can. J. Phys. 89, 429 (2011).

[18] K. T. Cheng, M. H. Chen, and W. R. Johnson, Phys. Rev. A 77, 052504 (2008).
[19] M. Andersson, Y. Zou, R. Hutton, and T. Brage, Phys. Rev. A 79, 032501 (2009).

[20] S. Schippers, D. Bernhardt, A. Müller, M. Lestinsky, M. Hahn, O. Novotný, D. W. Savin, M. Grieser, C. Krantz, R. Repnow, and A. Wolf, Phys. Rev. A 85, 012513 (2012).

[21] M. Hahn, D. Bernhardt, M. Lestinsky, A. Müller, O. Novotný, S. Schippers, A. Wolf, and D. W. Savin, Astrophys. J. 712, 1166 (2010).

[22] M. Hahn, D. Bernhardt, M. Grieser, C. Krantz, M. Lestinsky, A. Müller, O. Novotný, R. Repnow, S. Schippers, A. Wolf, and D. W. Savin, Astrophys. J. 729, 76 (2011).

[23] M. Hahn, M. Grieser, C. Krantz, M. Lestinsky, A. Müller, O. Novotný, R. Repnow, S. Schippers, A. Wolf, and D. W. Savin, Astrophys. J. 735, 105 (2011).

[24] K. Rinn, A. Müller, H. Eichenauer, and E. Salzborn, Rev. Sci. Instrum. 53, 829 (1982).

[25] M. Lestinsky et al., Astrophys. J. 698, 648 (2009).

[26] B. Hochadel, F. Albrecht, M. Grieser, D. Schwalm, E. Szmola, and A. Wolf, Nucl. Instr. Methods A 343, 401 (1994).

[27] K. Unser, IEEE Trans. Nucl. Sci. 28, 2344 (1981).

[28] Y. Ralchenko, A. Kramida, J. Reader, and N. A. Team, Nist Atomic Spectra Database (National Institute of Standards and Technology, Boulder, CO, 2011); [http://physics.nist.gov/asd].

[29] S. M. Younger, Phys. Rev. A 24, 1278 (1981).

[30] M. Arnaud and R. Rothenflug, Astron. Astrophys. Suppl. Ser. 60, 425 (1985)

[31] P. Mazzotta, G. Mazzitelli, S. Colafrancesco, and N. Vittorio, Astron. Astrophys. Suppl. Ser. 133, 403 (1998).

[32] S. Schippers, A. Müller, G. Gwinner, J. Linkemann, A. A. Saghiri, and A. Wolf, Astrophys. J. 555, 1027 (2001).

[33] S. M. Younger, Phys. Rev. A 22, 111 (1980).

[34] J. H. Macek and J. Botero, Phys. Rev. A 45, R8 (1992).

[35] K. P. Dere (2012, private communication).

[36] M. S. Pindzola, D. C. Griffin, and J. H. Macek, Phys. Rev. A 51, 2186 (1995). 